\title{
Assessment of computer game stressor on cognitive functions, parameters of stress and its effect on stress parameters after long term exercise
}

\author{
Mohita Singh ${ }^{1}$, Sunil Sachdev ${ }^{2}$, Amrita Singh ${ }^{3 *}$ \\ ${ }^{1}$ Senior Resident, ${ }^{2}$ Professor and Head, ${ }^{3}$ Senior Resident, ${ }^{1-3}$ Dept. of Physiology, ${ }^{1}$ Acharya Shri Chander College of Medical Sciences \\ Jammu, ${ }^{2,3}$ Government Medical College, Jammu, Jammu and Kashmir, India
}

*Corresponding Author: Amrita Singh

Email: amjohals@gmail.com

\begin{abstract}
Background: There are individual variations in response to same type of stress. Stress effects various parameters of higher mental functions such as attention, concentration, decision making, learning and memory. Stress is associated with decreased adaptability to life experiences and emotional maladjustment. The present study was designed to assess the effect of stressor on higher brain functions, stress parameters and effect of long term exercise on stress reactivity.

Materials and Methods: The study was conducted on thirty five healthy male volunteer students in two phases. In first phase of study, basal values and post stress values of cognitive functions and stress reactivity were assessed. In second phase of study, the subjects were again assessed after one month practice of moderate intensity physical exercise. Comparison was made on stress level between two phases. Result: In first phase, the values recorded for stress parameters including PR, SBP and DBP exhibited significant increase in value over pre stress values. Post stress result for serum cortisol was found to be non-significant. However, dual response was observed. Nineteen subjects exhibited significant increase and sixteen subjects exhibited significant decrease in post stress values over basal values. Post stress values significantly decreased for handgrip, RMT, ACT, STNT, STIT and STFT. Post stress values for ACS, STNS, STIS and STFS did not differ significantly. In second phase of study, results on stress parameters exhibited significant reduction in response and improvement in cognitive functions to stressor after one month practice of MIPE.
\end{abstract}

Conclusion: Stress response decreases after one month practice of MIPE.

Keywords: Stressor, Cognitive functions, Moderate intensity physical exercise, Stress response, Attention concentration span.

\section{Introduction}

Each individual responds differently to same type of stress due to unique genetic makeup and personal childhood experience with major stressors/trauma and personality characteristics. ${ }^{11}$ Stress effects various parameters of higher mental functions such as attention, concentration, decision making, learning and memory. ${ }^{21}$ Stress is associated with decreased adaptability to life experiences, negativity and maladjustment. ${ }^{8}$

The hormone cortisol increases glucose stores and makes energy available during stress. Glucocorticoids also form a second line of defence against stress by "suppression" of primary defence reactions. If corticosteroids fail to suppress it, the primary defence reactions themselves become damaging and the organism becomes susceptible to inflammatory and autoimmune diseases. If corticosterone levels are too extreme, a centrally regulated "drive" modulates HPA activity in order to maintain its set point. ${ }^{22}$

Regular exercise (thirty minutes of brisk walking everyday) induces persistent effects on cognition over the course of several months. ${ }^{3}$ Moderate to vigorous intensity exercise is an effective method for improving cognitive functions, perceived stress, stress symptoms and quality of life. $^{30}$

Stress is an inescapable part of life. Does stress effects higher functions of memory and attention concentration span? Does stress produces changes in physiological and biochemical parameters? Whether long term exercise improves stress response? To answer these intriguing questions, present study was designed to explore the effect of stressor and exercise on stress parameters and cognition.

\section{Materials and Methods}

The study was conducted on 35 healthy male volunteers chosen randomly within age group of $20-30$ years (mean \pm $\mathrm{SD}, 24.42 \pm 1.6$ ). Subjects with past or present significant history, psychiatric illness, CNS disorder, drug or alcohol abuse and any other known medical conditions were excluded. Only male subjects were chosen because females have different levels of stress and stress reactivity during different phases of menstrual cycle.

Ethical clearance for the study was taken from the Institutional Ethics Committee of Government Medical College Jammu, J\&K, India wide no. IEC /Pharma/Thesis /Research $/ \mathrm{T}_{7} \mathrm{~B} / \mathrm{C} / 2017 / 436$ dated 26/10/2017. The subjects were briefed about the study and informed written consent for participation was taken. Each subject served as his own control. Only educated volunteers were chosen for the reasons that adequate educational background is required for filling of questionnaires and visual time reaction measurements. All the experiments were done in forenoon to minimize the diurnal variations in cortisol level.

The study was performed in two phases. In the first phase of the study, basal parameters (cognitive functions and stress related parameters like BP, PR, handgrip, serum cortisol) were assessed. Computer game was introduced as a stressor and parameters were recorded during and in post stress period. In the second phase of the study, effect of one month practice of MIPE was assessed on stress parameters. 
Computer games usually give immense pleasure after a win. However, in the initial stages of the game when an individual suffers repeated defeats or constraints, the game turns very stressful. The stress that computer game gives was used as a stressor in the study. The physiological and psychological effects of computer game have already been successfully demonstrated. ${ }^{22}$

Serum cortisol is a well-known biochemical marker of stress and was assessed via ADVIA centaur cortisol assay. ${ }^{[28]}$ Isometric handgrip exercise is a resistance exercise that employs diastolic blood pressure. It is a measure of sympathetic system outflow for which handgrip dynamometer was used. ${ }^{[29]}$ Pulse rate was assessed via palpatory method by examining the radial artery. ${ }^{[5]}$ Blood pressure was measured with the help of sphygmomanometer by auscultatory method. Both systolic and diastolic blood pressure were recorded ${ }^{6}$ For assessing cognitive functions, Stroop colour and word interference test ${ }^{24}$ for cognitive flexibility; and PGI memory test ${ }^{18}$ for short term memory and attention concentration span were used in the present study.

Moderate intensity physical exercise: Subject were instructed to walk briskly at the rate of $4 \mathrm{mph} .{ }^{9}$ Subjects were instructed to carry out MIPE for 30 minutes a day for five days a week (150 minutes a week $)^{31}$ for one month duration.

\section{Statistical Analysis}

The statistical analysis was done using stats tester version 3.1.2. Comparison of pre-stress and post-stress mean data was done by student t-test. The results were computed as significant at $\mathrm{p}<0.05$ level $(*)$, more significant at $\mathrm{p}<0.01$ level $(* *)$ and highly significant at $\mathrm{p}<0.001$ level $(* * *)$.

\section{Results}

During stress, the values recorded for pulse rate, SBP and DBP exhibited significant increase in value over pre stress values (Table 11).

Post stress result for serum cortisol was found to be non-significant. However, dual response was observed. Nineteen subjects exhibited significant increase and sixteen subjects exhibited significant decrease in basal and post stress values. Post stress values significantly decreased for handgrip, RMT, ACT, STNT, STIT and STFT. Post stress values for ACS, STNS, STIS and STFS did not differ significantly (Table 2).

Table 1: Parameters analysed during stress

\begin{tabular}{|c|c|c|c|c|c|}
\hline S. No & Parameters & Basal Level & During Stress & p Value & Significance \\
\hline 1 & Pulse Rate & $76.74 \pm 4.63$ & $81.34 \pm 5.16$ & 0.0002 & $* * *$ \\
\hline 2 & $\mathrm{BP}$ & & & & \\
\hline (i) & SBP & $118.28 \pm 2.39$ & $121.71 \pm 3.21$ & 0.0000 & $* * *$ \\
\hline (ii) & DBP & $75.77 \pm 3.30$ & $77.71 \pm 3.21$ & 0.0165 & $*$ \\
\hline
\end{tabular}

PR: pulse rate (bpm); BP: blood pressure (mm Hg); SBP: systolic blood pressure; DBP: diastolic blood pressure

Table 2: Post stress parameters

\begin{tabular}{|c|c|c|c|c|c|}
\hline S. No & Parameters & Basal Level & Post Stress & p Value & Significance \\
\hline 1 & Handgrip & $24.51 \pm 4.09$ & $28.26 \pm 4.67$ & 0.0002 & $* * *$ \\
\hline 2 & Serum Cortisol & $12.48 \pm 4.21$ & $12.99 \pm 4.82$ & 0.6499 & NS \\
\hline (i) & Increase $(n=19)$ & $13.00 \pm 4.16$ & $15.92 \pm 4.19$ & 0.0433 & $*$ \\
\hline (ii) & Decrease $(n=16)$ & $12.37 \pm 4.07$ & $9.63 \pm 2.94$ & 0.0435 & $*$ \\
\hline 3 & Memory Test & & & & \\
\hline (i) & RMT & $23.73 \pm 4.24$ & $21.30 \pm 5.68$ & 0.0494 & $*$ \\
\hline (ii) & $\mathrm{AC}$ & & & & \\
\hline $\mathrm{a}$ & Score & $36.57 \pm 7.62$ & $35.62 \pm 8.73$ & 0.6368 & $\mathrm{NS}$ \\
\hline $\mathrm{b}$ & Time & $203.72 \pm 4.76$ & $201.38 \pm 4.89$ & 0.0495 & $*$ \\
\hline 4 & Stroop test & & & & \\
\hline (i) & Neutral & & & & \\
\hline $\mathrm{a}$ & Score & $64.51 \pm 6.66$ & $66.85 \pm 5.27$ & 0.1128 & $\mathrm{NS}$ \\
\hline $\mathrm{b}$ & Time & $841.17 \pm 130.94$ & $737.57 \pm 80.31$ & 0.0002 & $* * *$ \\
\hline (ii) & Interference & & & & \\
\hline $\mathrm{a}$ & Score & $73.57 \pm 3.81$ & $74.77 \pm 3.78$ & 0.1968 & NS \\
\hline $\mathrm{b}$ & Time & $900.88 \pm 92.36$ & $850.34 \pm 101.21$ & 0.0350 & $*$ \\
\hline (iii) & Facilitation & & & & \\
\hline $\mathrm{a}$ & Score & $77.62 \pm 3.23$ & $78.34 \pm 3.54$ & 0.3885 & NS \\
\hline $\mathrm{b}$ & Time & $746.22 \pm 125.45$ & $683.91 \pm 124.66$ & 0.043 & $*$ \\
\hline
\end{tabular}

IHG: isometric handgrip (mm Hg); S. Cort: serum cortisol $(\mu \mathrm{g} / \mathrm{dl})$; RMT: recent memory time; ACS: attention concentration score; ACT: attention concentration time. Memory test time is measured in seconds. STNS: stroop test neutral score; STNT: 
stroop test neutral time; STIS: stroop test interference score; STIT: stroop test interference time; STFS: stroop test facilitation score; STFT: stroop test facilitation time. Stroop test time is measured in milliseconds.

Effect of stressor after one month practice of MIPE

Results of the effect of stressor on all stress parameters after one month practice of exercise was observed. Pulse rate, SBP, handgrip recorded significant increase while DBP and serum cortisol remained non-significant. However serum cortisol exhibited dual response. While ten subjects exhibited significant increase, twenty five subjects exhibited significant decrease in serum levels in pre and post stress phase. The response to stressor was lower after one month practice of MIPE compared to first phase of the study (Table 3).

Table 3: Effect of stressor after one month practice of MIPE

\begin{tabular}{|l|c|c|c|c|c|}
\hline S. No & Parameters & Pre-stress Value & Post-stress value & p Value & Significance \\
\hline 1 & Pulse rate & $72.74 \pm 4.83$ & $75.74 \pm 5.11$ & 0.0154 & $* *$ \\
\hline 2 & BP & & & & \\
\hline (i) & SBP & $116.68 \pm 1.96$ & $118.22 \pm 1.95$ & 0.0018 & $* *$ \\
\hline (ii) & DBP & $73.37 \pm 3.30$ & $74.97 \pm 3.58$ & 0.0600 & NS \\
\hline 3 & Handgrip & $21.08 \pm 3.39$ & $23.94 \pm 3.89$ & 0.0019 & $* *$ \\
\hline 4 & S. Cortisol & $12.48 \pm 4.19$ & $11.32 \pm 3.91$ & 0.1737 & NS \\
\hline (i) & Increase ( $=10)$ & $11.68 \pm 2.79$ & $15.25 \pm 3.73$ & 0.0337 & $*$ \\
\hline (ii) & Decrease (n=25) & $12.80 \pm 4.60$ & $9.75 \pm 2.68$ & 0.0072 & $* *$ \\
\hline
\end{tabular}

\section{Discussion}

In modern times of globalization and enhanced performance, none is escaped from stress. Stressful life events and daily life stresses have both deleterious and cumulative effects on human body. Several studies have reported that stress affects various parameters of higher mental function like attention, concentration, learning and memory. Generally all stressful events generate certain category of emotions of varying intensity which may also affect cognition and performance.

Computer game acts as an acute stressor. The computer game stressor was more close to daily life stresses instead of symptoms of traumatic stress. The physiological and psychological effects of stress with the use of computer game to stimulate the effect of acute psychological stress have been demonstrated. ${ }^{22}$

\section{Effect of stress}

In present study, PR, SBP and DBP during stress showed significant increase over basal level. This increase in post stress value is positive health outcome to cope up with the stress level which is in corroboration with the earlier studies. ${ }^{26}$ Body releases adrenaline in stressful situations, a hormone that temporarily causes increase in heart rate and blood pressure. These reactions prepare the body to deal with the situation — the "fight or flight" response. $^{17}$

The increase in handgrip value (requiring DBP measurement) in post stress period was observed in similar studies in response to isometric handgrip exercise. ${ }^{7}$ Isometric handgrip exercise provides pressure stimuli to cardiovascular system through efferent sympathetic pathway with the resultant increase in heart rate and BP. ${ }^{15}$

Serum cortisol, commonly considered a biochemical marker of stress, exhibited diabolic response with 19 subjects observed significant increase and 16 subjects by significant decrease in levels. Similar responses of both increase and decrease in cortisol concentration after computer game stressor were observed. ${ }^{10,14}$ The body response towards stress challenges occur to bring back the body to a state of physiological and psychological homeostasis. Slower response to stress is characterized by release of cortisol and may take hours for adaptive effects to emerge. This occurs via activation of HPA axis through increase of CRF level in hypothalamus leading to increase in $\mathrm{ACTH}$ level from anterior pituitary that triggers glucocorticoids secretions from adrenal cortex. These steroids bind with GR and MR receptors and thus producing response by acting at DNA level. ${ }^{27}$

There was no significant effect after stress on memory and stroop test scores while memory and stroop test time decreased significantly in post-stress period showing enhancement of performance. Similar results of significant reduction in memory time and reaction time related to stress were observed. ${ }^{23}$ Cahill $\mathrm{L}^{1}$ studied the well-known beneficial effect of stress on cognitive behaviours as represented by the mid portion of inverted $U$ shape relationship between stress and performance. Enhanced memory associated with emotional arousal results from an activation of beta-adrenergic stress hormone system during and after an emotional experience.

Effect of stress after one month practice of MIPE

After one month of moderate intensity physical exercise, response to stressor was lower compared to basal values. PR, SBP and DBP reported significant decrease which is in corroboration to earlier studies. ${ }^{19,12}$ Handgrip value exhibited significant decrease in response. Pagonas ${ }^{16}$ in there study comparing long term aerobic exercise and isometric handgrip exercise stated that aerobic exercises led to significant reduction in DBP while no statistical significant changes were recorded with isometric handgrip exercise, proving that reduction in DBP and hence handgrip values in present study was due to effect of aerobic exercise. Serum cortisol level reported non-significant changes with 
tendency to decrease. Regular exercise modulates the response of cortisol elevation over time and lead to adaptation of endocrine system to exercise training. ${ }^{4}$

A study with larger sample size is needed for assessing the effect of computer game stressor on stress parameters and cognitive functions. The results of the study maybe specific to the type of subject chosen and individual variation in intelligence to handle stressful conditions.

\section{Conclusion}

Computer game stressor produce physiological changes in body to cope with stressful situations. It is marked by increase in sympathetic outflow in response to stress. Tendency of serum cortisol to increase, a biochemical marker of stress, differ at individual level due to variation in intelligence to handle stressful situations. Improvement in cognitive functions after the stressor indicates stress induced increase in arousal and vigilance.

After one month practice of MIPE, decrease in stress response to stressor indicates that exercise on long term basis reduces stress reactivity. Tendency of serum cortisol level to decrease indicates down-regulation of sympathetic system with long term exercise. Stressful conditions leads to changes in physiological and biochemical parameters to bring back the body to a state of homeostasis.

\section{Acknowledgement}

This work is an original article and no financial grants were obtained from any source. There is no conflict of interests.

\section{Conflict of Interest: None.}

\section{References}

1. Cahill L, Prins B, Weber M, McGaugh JL. Beta adrenergic activation and memory for emotional events. Natur 1994;371:702-4

2. Cassens G, Roffman G, Kuruc A, Orsulak PJ, Schildkraut JJ. Alteration in brain norepinephrine metabolism induced by environmental stimuli previously paired with inescapable shock. Sci 1980;209:1138-9.

3. Cox EP, O’Dwyer N, Cook R, Vetter M, Cheng HL, Rooney $\mathrm{K}$, et al. Relationship between physical activity and cognitive function in apparently healthy young to middle aged adults: A systematic review. J Sci Med Sport 2016;19(8): 616-28

4. Dallman MF, Akana SF, Levin N, Walker CD, Bradburg MJ, Suemaru S, et al. Corticosteroids and control of function in HPA axis. Ann N Y Acad Sci 1994;746:22-31

5. Ghai CL. Clinical examination of the cardiovascular system. In Ghai CL (ed): A textbook of medical physiology. $8^{\text {th }}$ Edition, Jaypee Bros Med Publ 2013:264-65.

6. Ghai CL. Recording of systemic arterial blood pressure. In Ghai CL (ed): A textbook of practical physiology. $8^{\text {th }}$ Edition, Jaypee Bros Med Publ 2013: pp 171-3.

7. Goel A, Ghosh S, Dhar U, Shrivastav R, Krishana B. Effect of examination stress on cardiovascular reactivity to isometric handgrip exercise in first year medical students. Intl J Physiol 2016; 4(2): 174-179

8. Gorban AN, Tyukina TA, Smirnova EV, Pokidysheva LI. Evolution of adaptation mechanisms: Adaptation energy, stress and oscillating death. $J$ Theor Biol 2016; 405:127-139

9. Haskell LW, Lee MI, Pate RR, Powell EK, Blair NS, Franklin AB, et al. Physical Activity and Public Health Updated
Recommendation for Adults from the American College of Sports Medicine and the American Heart Association. Circu 2007;116:1081-93

10. Haussman MF, Vleck CM, Farrar ES. A laboratory exercise to illustrate increased salivary cortisol in response to three stressful conditions using competitive ELISA. Advan Physiol Educ 2007;31:110-15

11. Jeronimus BF, Ormel J, Aleman A, Penninx BWJH, Riese H. Negative and positive life events are associated with small but lasting change in neuroticism. Psychol Med 2013;43(11):240315

12. Kelley GA, Kelly KS. Progressive resistance exercise and resting BP: A meta-analysis of randomized controlled trials Hypertens 2000;35:838-43.

13. Korf J, Aghajanian GK, Roth R. Increased turnover of norepinephrine in the rat vertebral cortex during stress: Role of locus coeruleus. Neuropharmacol 1973;12:933-8.

14. Loft P, Thomas MG, Petrie KJ, Booth RJ, Miles J, Vedharad K et al. Examination stress results in altered cardiovascular responses to acute challenge and lower cortisol. Psychoneuroendocrinol 2007;32:367-75.

15. Mathias CJ, Bannister R. Investigation of autonomic disorders. In Bannister R, Mathias CJ (eds): Autonomic failure, a textbook of clinical disorders of ANS. Oxfor Univ Press 1992:266.

16. Pagonas N, Vlatsas S, Bauer F, Seibert FS, Zidek W, Babel N, et al. Aerobic versus isometric handgrip exercise in hypertension: A randomized controlled trial. J Hypertens 2017;35(11):2199-2206.

17. Pal GK, Pal P, Nanda N. Autonomic nervous system. In Shree A, Vohra CM (eds): Comprehensive textbook of medical physiology. $1^{\text {st }}$ Edition, Auth Jaypee Bros Med Publ 2017;1:299-300.

18. Pershad D, Wig NN. Reliability and validity of a new battery of memory tests (PGI Memory Scale). Ind J Psy 1978; 20: 7680.

19. Rennie KL. Effects of moderate and vigorous physical activity on heart rate variability in a British study of civil servants. Am J Epidemiol 2003;158(2):135-43

20. Romero LM, Sapolsky RM. Patterns of ACTH secretagogue secretions in response to psychological stimuli. $J$ Neuroendocrinol 1996;8:243-58.

21. Sahin NH, Guler M, Basim HN. The Relationship between Cognitive Intelligence, Emotional Intelligence, Coping and Stress Symptoms in the context of Type A Personality Pattern. Turk J Psy 2009;20(3):243-54.

22. Sharma R, Khera S, Mohan A, Gupta N, Ray RB. Assessment of computer game as a psychological stressor. Ind J Physiol Pharmacol 2006;50(4):367-74.

23. Singh Y, Sharma R. Relationship between general intelligence, emotional intelligence and stress level and stress reactivity. $J$ Ind Acad Neurosci 2012;19(3):107-111.

24. Siska E. The Stroop colour word test in psychology and biomedicine. Acta Univ Palacki Olomuc Gymn 2002;32(1):4552.

25. Svensson TH. Peripheral, autonomic regulation of locus coeruleus noradrenergic neuron in the brain putative implications for psychiatry and psychopharmacology. Psychopharmacol 1987;92:1-7

26. Trapp M, Trapp EM, Egger JW, Domej W, Schillaci G, Avian A, et al. Impact of mental and physical stress on BP and pulse pressure under normobaric versus hypoxic conditions. Plos One 2014;9(5):1-21

27. Trentani TA, Kuipers SD, Ter Horst GJ, Den Boer JA. Selective chronic stress induced in vivo ERK1/2 hyperphosphorylation in medial prefrontocortical dendrites: Implications for stress related cortical pathology? Eur J Neurosci 2002;15:1681-91 
28. US Food and Drug Administration. Siemens centaur cortisol assay. Siemens HC 2012: pp 1-12, USA

29. Van den Berg MP, Smit AJ. Bedside autonomic function testing in patients with vasovagal syncope. Pace Clin Electrophysiol 1997;20:2039-42.

30. Wilcox S, Dowda M, Leviton LC, Bartlett-Prescott J, Bazzarre T, Campbell-Voytal K et al. Active for life-final results from the translation of two physical activity programs. Am J Prev Med 2008;35(4):340-45
31. World Health Organization. The Global Recommendations on Physical Activity for Health. WHO Press 2010:16, 26, Geneva, Switzerland

How to cite this article: Singh M, Sachdev S, Singh A. Assessment of computer game stressor on cognitive functions, parameters of stress and its effect on stress parameters after long term exercise. India $J$ Neurosci 2019;5(2):64-8. 